\title{
Science at mid-infrared wavelengths with large telescopes
}

Charles M. Telesco, David Ciardi, Chris C. Packham

Charles M. Telesco, David Ciardi, Chris C. Packham, "Science at mid-infrared wavelengths with large telescopes," Proc. SPIE 4834, Discoveries and Research Prospects from 6- to 10-Meter-Class Telescopes II, (13 February 2003); doi: 10.1117/12.460956

SPIE Event: Astronomical Telescopes and Instrumentation, 2002, Waikoloa, Hawai'i, United States 


\title{
Science at mid-infrared wavelengths with large telescopes
}

\author{
C. M. Telesco*, D. Ciardi*, C. Packham* \\ Department of Astronomy, University of Florida
}

\begin{abstract}
For background-limited observations such as those at mid-infrared wavelengths, an increase in telescope aperture not only improves the angular resolution (as it does at all wavelengths) but also leads to very large increases in sensitivity because of the reduced background within the diffraction-limited point spread function. In this presentation we look at the magnitude of that increase for several different operational modes of mid-infrared instrumentation, and we consider some of the implications for science on the current generation of 8-10 meter telescopes.
\end{abstract}

Keywords: infrared imaging, spectroscopy, polarimetry, coronagraphy

\section{THE MID-INFRARED: PROMISES AND CHALLENGES}

The high ambient thermal background emission in the mid-infrared (5-30 microns) usually ensures that, with state-ofthe-art detectors, background shot noise is the limiting noise at these wavelengths. For background-noise-limited observations, an increase in telescope aperture not only improves the angular resolution, but also leads to very large increases in sensitivity because of the lower background coincident with the smaller diffraction-limited point spread function. The signal-to-noise ratio achieved in an integration time $t$ for a point source observed with a telescope of aperture diameter $D$ is proportional to $D^{2} t^{1 / 2}$. More interestingly, the integration time it takes to achieve a given signal-tonoise ratio is inversely proportional to $D^{4}$. To get the same point-source sensitivity on a 4-meter telescope provided by five $\mathrm{h}$ of integration at an 8-meter telescope (which, with overheads, is essentially a full night of observing) would require $80 \mathrm{~h}$ of 4 -meter integration time. With normal operational overheads, this difference amounts to 1 observing night on the 8-m compared to at least eight nights on the 4-m. This remarkable gain and, of course, the increase in angular resolution, per se, indicate that the new generation of large telescopes are able to provide us with a uniquely powerful probe of the mid-infrared universe. There is one important caveat, though: the achievable signal-to-noise ratio is also inversely proportional to $\varepsilon^{1 / 2}$, where $\varepsilon$ is the emissivity along the optical path, and so the sensitivity gains of a large telescope aperture can be lost if the telescope emissivity is compromised. For example, an increase in emissivity from $3 \%$ (Gemini's goal) to $12 \%$ for a 10-meter telescope results in its having a point-source sensitivity equivalent to a 7 -meter telescope with an emissivity of $3 \%$.

From high, dry sites, there are two excellent atmospheric windows, one near $10 \mu \mathrm{m}$ and one near $20 \mu \mathrm{m}$, which permit mid-infrared observations to be made with large groundbased telescopes. When considering the mid-infrared science that these telescopes can uniquely advance, it is helpful to keep in mind their role in relation to mid-infrared space telescopes, particularly SIRTF, which is imminent. SIRTF's mid-infrared sensitivity will be a hundred times better than those of Gemini or the GTC, and of course some spectral regions inaccessible from the ground will be open to SIRTF. However, the angular resolution from the ground will be more than an order of magnitude better than SIRTF's. Thus, the large groundbased telescopes will be uniquely incisive probes of angular structure smaller than a few arcseconds, and they can provide spectroscopic and other capabilities not supported by SIRTF and other space facilities. In this presentation we consider several science programs that illustrate the performance and promise of groundbased 8 and 10$\mathrm{m}$ telescopes at mid-infrared wavelengths.

\section{BROWN DWARFS}

Brown dwarfs (BDs) have masses between those of stars and planets. The upper mass limit is about 0.08 solar masses (about 75 Jupiter masses), with the lower boundary being somewhat more poorly defined but being near 10 Jupiter masses ${ }^{1}$. They are distinguished from stars by their not being structurally stabilized by nuclear fusion, although they do experience a short phase of deuterium burning. After nearly a decade of searching by many observers, the detection of Gliese 229B in 1995 identified the object first generally accepted to be a brown dwarf ${ }^{2}$. Since then, as a result of a 
variety of optical and near-IR surveys, the number of known BDs has grown to about 100. Optical and near-IR photometric and, particularly, spectroscopic observations of this rapidly increasing sample are supporting an intense effort to model their structure and atmospheres. Mid-infrared observations can provide valuable additional information to further our understanding of these objects.

Because we have a fair amount of information about Gl 229B, it is a useful example of the BD class. In Fig. 1 we present the synthetic (model) spectral energy distribution of $\mathrm{Gl} 229 \mathrm{~B}^{3}$, which is $5.7 \mathrm{pc}$ away, has an effective temperature of about $1000 \mathrm{~K}$, and is now classified as a member of the new spectral class of $\mathrm{T}$ dwarfs ${ }^{4}$. Gl $229 \mathrm{~B}$ is 44 AU away in projection from the M1 main sequence star GL 229A, which contributes negligibly to the heating of Gl 229B. The BD's average flux density across the $10-\mu \mathrm{m}$ window is about $2 \mathrm{mJy}$. The rectangles in the figure correspond to photometric detections ${ }^{5}$. The point-source broadband sensitivities of current and planned 8-10 meter telescopes are typically about $0.05 \mathrm{mJy}(\mathrm{S} / \mathrm{N}=1$ in 1 hour of chopped integration). A BD like Gl 229B is easy to detect. If we are willing to integrate for several hours, which, with observational overheads, corresponds to a full night of telescope time, we can detect a source like Gl 229B in broadband imaging with $\mathrm{S} / \mathrm{N}=5$ located as far away as about 25 pc. Thus, we could straightforwardly detect by broadband imaging at $10 \mu \mathrm{m}$ about $1 / 3$ of the sample of $\mathrm{L}$ and $\mathrm{T}$ dwarfs presented by Kirkpatrick et al. ${ }^{4}$, if they had mid-IR luminosities comparable to that of Gl 229B. For many types of observations of isolated, single brown dwarfs found in time to be observed by SIRTF, it would be more efficient for them to be observed by SIRTF. However, the big advantages of using large groundbased telescopes for these objects will be (1) to observe objects not found early enough for them to be observed by SIRTF, (2) to determine the characteristics of the environments very near the BDs, with particular attention being paid to issues such as multiplicity and formation that require high angular resolution, and (3) to achieve higher spectral resolutions or apply observational techniques not supported by SIRTF.

The second of these three advantages is illustrated by considering the SIRTF Legacy Science Program "From Molecular Cores to Planet-Forming Disks" (Neal Evans, Principal Investigator). As part of that program they will map at several wavelengths roughly 10 square degrees of the Perseus molecular cloud, which is $300 \mathrm{pc}$ away. They are likely to find scores of young stellar and sub-stellar objects that are heating local dust that then emits detectable mid-infrared radiation. The big telescopes will be particularly useful in probing those stellar and BD birthplaces with as good as 0.2 0.3 arcsec resolution, a significant advantage since they are sure to have interesting structure at sub-arcsec scales unresolvable by SIRTF. Furthermore, as illustrated in Fig. 2 (adapted from N. Evans, link at SIRTF Web site), dusty envelopes and disks heated by the BDs should be easily detectable in the mid-infrared for sources even as far away as

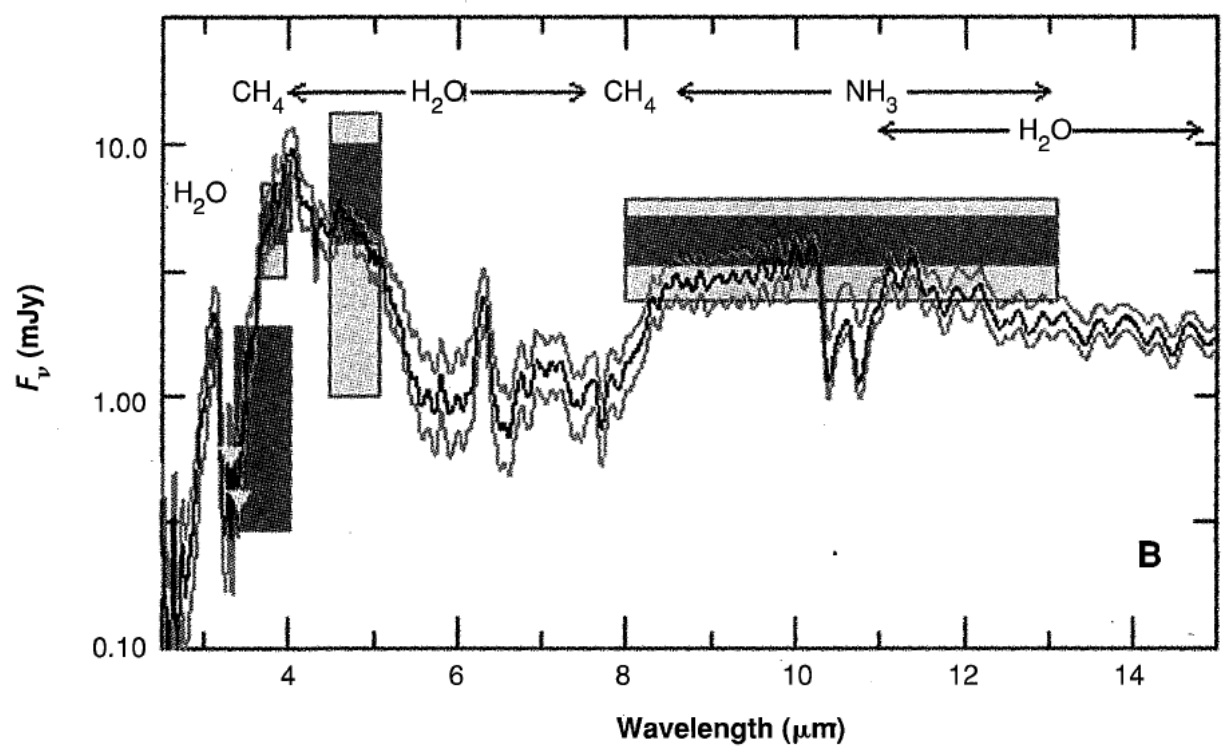

Fig. 1: The mid-infrared spectrum predicted for GL $229 \mathrm{~B}^{3}$. 


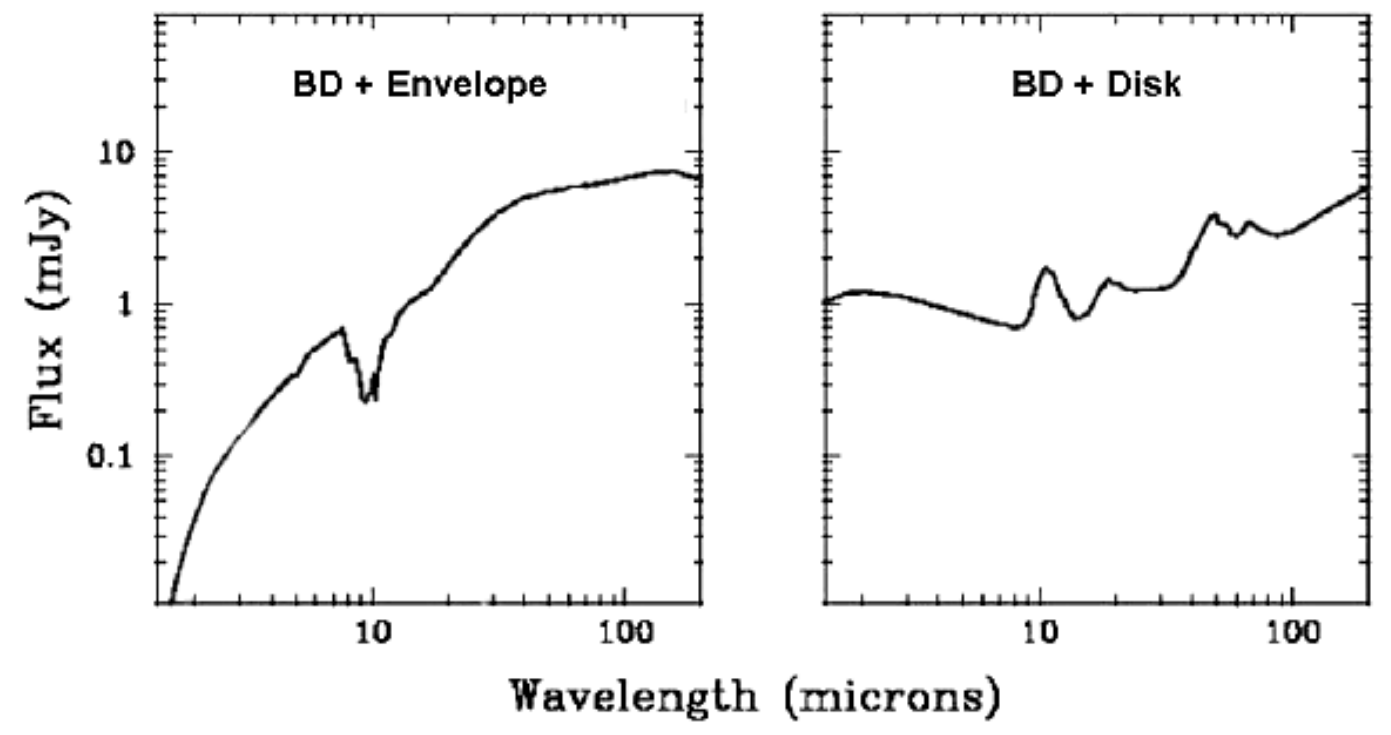

Fig. 2: SED for BD sources in the Perseus cloud.

300 pc. The recent detection ${ }^{6}$ (Fig. 3) of bright excess $10 \mu \mathrm{m}$ emission at a level of about $20 \mathrm{mJy}$ associated with the BD candidate Chamaeleon $\mathrm{H} \alpha 2$ (distance about $150 \mathrm{pc}$ ) shows that this is indeed a reasonable expectation, and it indicates the kind of analysis that even simple photometric measurements can support. The level of the excess emission is that expected for an optically thick flat disk, rather than a flared disk.

Coronagraphic searches for BD companions to stars require high angular resolution and can be done effectively with large groundbased telescopes, although this is currently an unexplored technique at mid-infrared wavelengths. The GTC instrument CanariCam being developed for the GTC has a coronagraphic mode described in more detail in another contribution to this conference. To illustrate the gains anticipated for this mode we consider the baseline CanariCam coronagraphic mode under optimal atmospheric conditions. In this configuration a 0.8 arcsec-radius, hard-edged (top hat) occulting spot is placed at the telescope focal plane within the instrument, and a rotating pupil mask that blocks the central secondary-mirror obscuration and approximately matches the hexagonal shape of the primary is placed at a pupil image. The secondary-mirror spider images are masked at the pupil, but the edges of the mirror segments are not. At a distance of 1 arcsec or greater from the star, the stellar point spread function is suppressed by about an order of magnitude.

To detect a faint companion around the occulted star one subtracts from the program star's observed intensity profile that of a comparison star observed in the same coronagraphic configuration. This subtraction of the normalized profile can be made to an $m$ (fractional) level of accuracy (i.e., probable error equals $m$ times the intensity at the radius where the companion may be located). Experience in the near infrared suggests that $m$ is approximately 0.1. Therefore, if the coronagraphically suppressed profile is about ten times fainter than the standard (non-coronagraphic) imaging profile, the absolute error in the measured flux is about ten times smaller than for standard imaging. These uncertainties represent noise floors, which, once they have been achieved through an appropriate integration time are not likely to be improved upon. The integration time is set so that the thermal-background shot noise is small compared to the absolute error fixed by $m$. For example, assuming that $m=0.1$, the $1-\sigma$ detection limit for an object located $10 \mathrm{AU}(1 \mathrm{arcsec})$ from a Sun-like star $10 \mathrm{pc}$ away is 150 microJanskys in the standard (non-coronagraphic) imaging mode; i.e., the intensity in the stellar profile 1 arcsec from the peak is one thousand times fainter than the peak value, and therefore a companion with a flux a thousand times fainter than the star will have a peak intensity equal to the star's intensity at 1 arcsec radius. The process of subtracting a calibration profile (obtained using another point source) from the stellar profile leaves a residual (noise) about ten times fainter than this (150 microJanskys), corresponding to the value $m=1$. Because of the order of magnitude suppression ratio for the coronagraphic mode, the same line of reasoning indicates that the $1-\sigma$ detection limit is about 15 microJanskys in the coronagraphic mode. A chopped integration time of 4 hours 


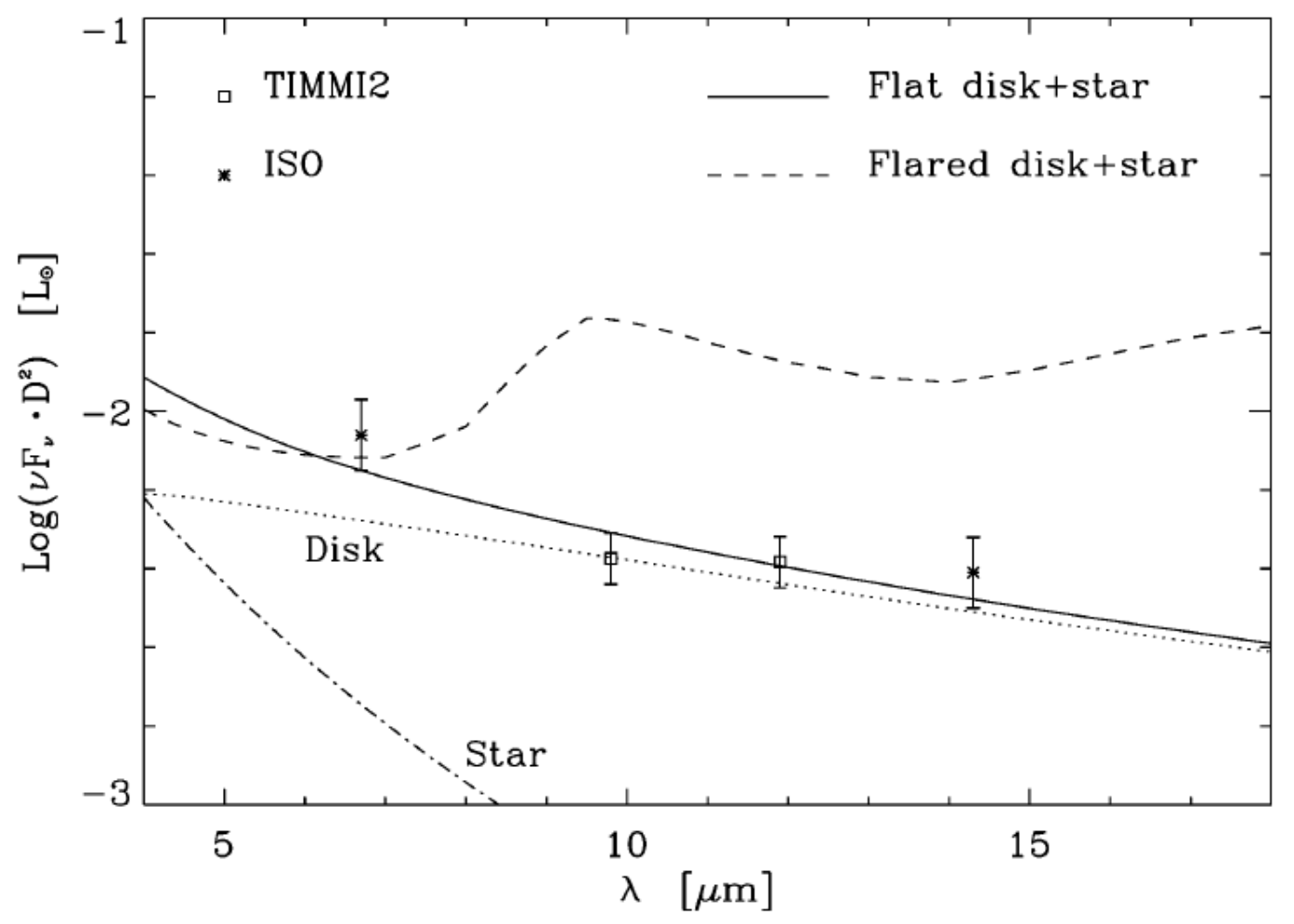

Fig. 3: Model spectral energy distributions of a BD ("star") with an optically thick flat disk and a flared disk, compared to observations of Chamaeleon $\mathrm{H} \alpha 2^{6}$.

on the program source plus a comparable amount of time on the profile-comparison standard would provide a $1-\sigma$ noise level of about 30 microJanskys, a factor of two worse than the potential limit set by the coronagraphic mode, but much better that the sensitivity for faint companions set by profile subtraction in the standard imaging mode. These gains may be partly overshadowed by other systematic effects that will be explored with CanariCam at the GTC, but the potential for this mode is very high.

To illustrate the coronagraphic mode further, we consider a Gl 229B-type BD at 10 AU from a G2V star located 10 pc away. The $10 \mu \mathrm{m}$ flux densities of the star and the BD are $1.5 \mathrm{Jy}$ and 650 microJansky, respectively. Using the above line of reasoning, in the standard imaging mode the signal-to-noise ratio at the position of the BD would be limited to a value of about 4.3, which could be achieved in $1 \mathrm{~h}$ of chopped integration on the source and another hour on the comparably bright point-spread-function calibrator. Integrating longer does not improve that detection. Using the baseline coronagraphic mode on CanariCam, the limiting signal-to-noise ratio is about 43 . By integrating for 4 hours on the program source and the same time on the PSF-comparison star, one can detect the BD with a signal-to-noise ratio of about 25 .

A broad variety of astrophysically significant diagnostics are apparent in the mid-infrared and accessible with low-tomoderate resolution spectroscopy. The model spectrum of Gl 229B (Fig. 1) illustrates this point for brown dwarfs. Gemini, the GTC, and the VLT could obtain mid-infrared spectra of GL 229B with a spectral resolution of 50 with a signal-to-noise ratio of about three in $1 \mathrm{~h}$ of integration.

\section{CIRCUMSTELLAR DISKS}

Planets are thought to form in dusty circumstellar disks, and so the exploration of disks contributes fundamentally to a broader understanding of the origin and evolution of our own and other planetary systems. One expects the detailed structure (e.g., size, shape, and density) of a very young disk to markedly influence how and where planets are formed 
there. On the other hand, the detailed structure of an older, more evolved disk should bear traces of its history and of any planets that may have already formed, or are in the process of forming. In one widely accepted scenario, gas and dust accrete directly onto the central forming protostar during the initial phase of cloud collapse. Eventually, the primary accretion shifts to a circumstellar disk, which accumulates enough mass $(\sim 0.3$ of the stellar mass) to become gravitationally unstable, form spiral arms, and transport angular momentum outward. At this point, the disk is the main source of matter accreting onto the star. As the protocloud is depleted, accretion onto the disk ceases, and both the disk mass and the accretion rate onto the star decrease. Stellar winds, UV radiation, planetesimal/planet formation, and residual accretion completely erode the primordial disk, which is replaced by a more diffuse "debris disk" that is continually replenished with comet dust and collisional fragments.

It has been shown that disks can be strong sources of mid-infrared radiation emitted by dust heated by starlight and/or (for the densest, youngest disks) viscous accretion. Where heating by starlight dominates, we expect a monotonic falloff of the dust temperature with distance from the star. Generally in astrophysical environments the dust we see in the midinfrared is hotter than about $100 \mathrm{~K}$, temperatures that are attained within a hundred or so AU of an A star. This scale is comparable to the size of our solar system, so the mid-infrared emission from disks is a particularly useful probe of circumstellar regions relevant to the exploration of the origin and evolution of planets. The diffraction limits of 8-m and $10-\mathrm{m}$ telescopes are about $0.2 \operatorname{arcsec}$ and $0.4 \operatorname{arcsec}$ at 10 and $20 \mu \mathrm{m}$, respectively. Thus, for a star at $20 \mathrm{pc}$, we can resolve details in disks that have scale sizes of the order of 5-10 AU, clearly useful for the exploration of planetary disks. We also add that, as illustrated by the computed spectral energy distribution shown in Figure 3, disks are expected to be apparent at mid-infrared wavelengths around brown dwarfs, although they are unlikely to be large enough to be resolvable.

We all know that you get better angular resolution as you go to a larger telescope, but the following illustration demonstrates how much additional information even a factor of a little more than two can provide. Fig. 4 (left panel) shows the $18 \mu \mathrm{m}$ image made with the mid-infrared camera OSCIR at the CTIO 4-m telescope of the circumstellar disk around the star HR $4796 \mathrm{~A}^{7}$. The nearly edge-on disk is clearly evident. Using the same camera at the same wavelength on the $10-\mathrm{m}$ Keck 2 telescope provided the image shown in the right panel of the same figure ${ }^{8}$. In the latter image one clearly sees three blobs: the central one is the stellar photosphere, and the outer ones are the edge-on disk seen in the emission from its dust. This image was used to provide a basis for detailed dynamical modeling of the disk that could be related to the disks evolution and the possible gravitational effects of the companion star and/or planets located in the inner region.

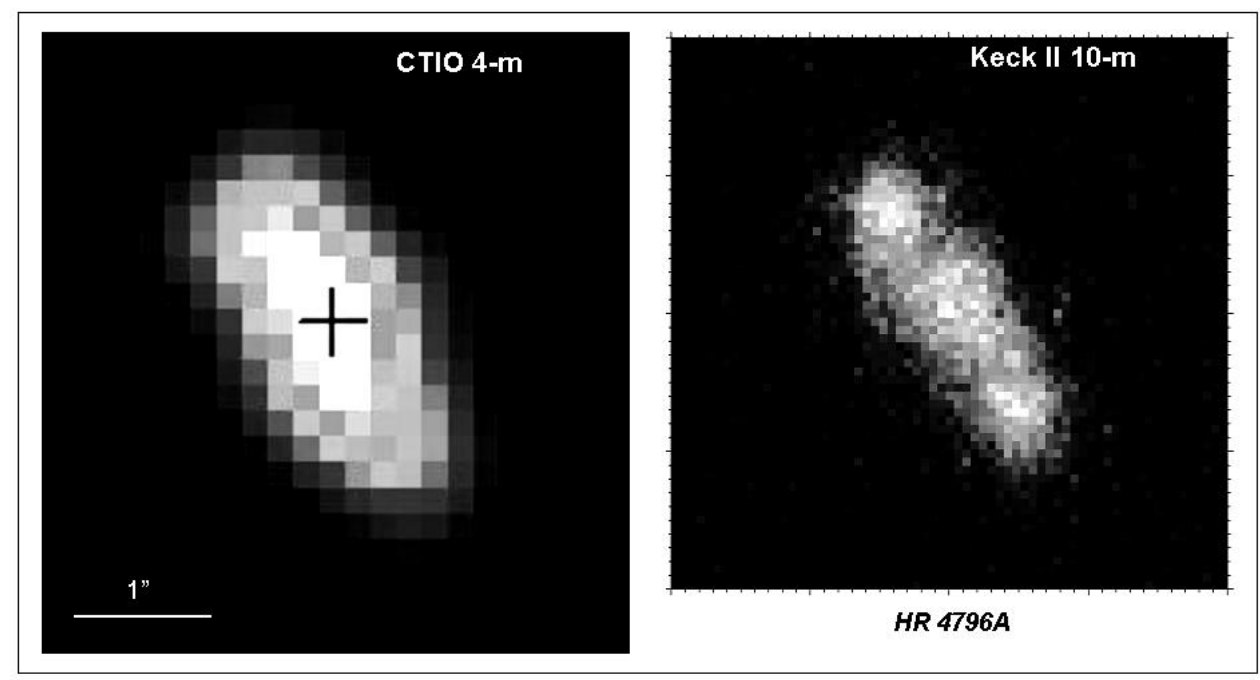

Fig. 4-OSCIR images at $18 \mu \mathrm{m}$ of the edge-on circumstellar disk associated with HR 4796A. Image at left made with the CTIO 4-m Blanco telescope ${ }^{7}$. Image at right made at Keck $2^{8}$. 


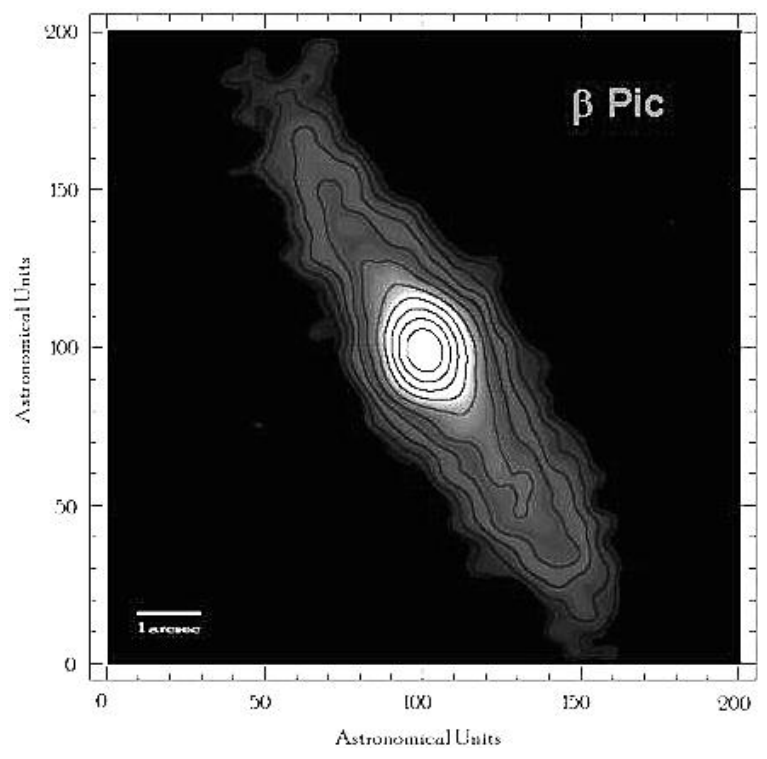

Fig. 5: The edge-on debris disk around $\beta$ Pictoris, as imaged at $18 \mu \mathrm{m}$ with OSCIR using Gemini South ${ }^{9}$.

The circumstellar disk around the main sequence A star $\beta$ Pictoris is the most well known example of this phenomenon. $\beta$ Pic's disk, which is known to be viewed edge on, is designated a "debris disk." Disks around main sequence stars are called Vega-type disks or debris disks, the latter designation indicating that generally these systems are too old for the dust to be primordial. The disk observed near $20 \mu \mathrm{m}$, as presented in Fig. $5^{9}$, is really the inner few hundred AU of a much large disk extending out to nearly a thousand AU. These outer regions are so cool, however, that they are only apparent at far infrared-through-millimeter wavelengths. The inner region observed with high resolution in the midinfrared exhibits many features that may be due to the presence of planets. As the image in Fig. 5 shows, ripples and other brightness asymmetries are obvious. The dynamical effects of a giant planet in a somewhat inclined orbit may be account for some of these asymmetries.

\section{STAR FORMING REGIONS}

The well-established complexity of star forming regions requires probing with high angular resolution to minimize confusion and ambiguity. A good example of the gains achieved by large telescopes is the recent Gemini imaging of the young stellar source L1448-IRS3, which is also located in the Perseus cloud considered above. L1448-IRS3 consists of three sources (designated A, B, and C) that have been resolved at millimeter wavelengths ${ }^{10}$. Sources A and B, which, together, have previously been classified as a class 0 source, are separated from each other by 7" (2100 AU), with C being roughly 20" away from these two. A class 0 source is regarded as the earliest phase-the protostellar phase-of star formation in which the mass of the infalling envelope still exceeds that of the central star. These objects have been elusive, and so the high-angular-resolution exploration of any candidates is critical. In fact, it has been suggested that L1448-IRS3 sources A and B may constitute a protostellar class 0 binary system in a common envelope. They have been unresolved for wavelengths shorter than $2.7 \mathrm{~mm}$, so the primary sources of the luminosity (10.9 solar luminosities) and the nature of the individual sources have been unknown.

Recent mid-infrared imaging (at 10, 18, and $24 \mu \mathrm{m}$ ) of L1448-IRS3 centered on components A and B have been made by Ciardi et al. ${ }^{11}$ using Gemini North. The $24 \mu \mathrm{m}$ image is shown in Fig. 6. These images show that virtually all of the 10-30 $\mu \mathrm{m}$ emission arises in component A, which suggests that component B's envelope is denser and more optically thick than that of A. This in turn suggests that A may be more like a class 1 young stellar object than a class 0 object, whereas B may have the denser, more optically thick envelope expected for a class 0 source. This conclusion is consistent with the large envelope mass determined for B from millimeter observations. Although these sources (A, B, and C) could have been detected and resolved with a smaller telescope, it is evident that more distant, more compact, 

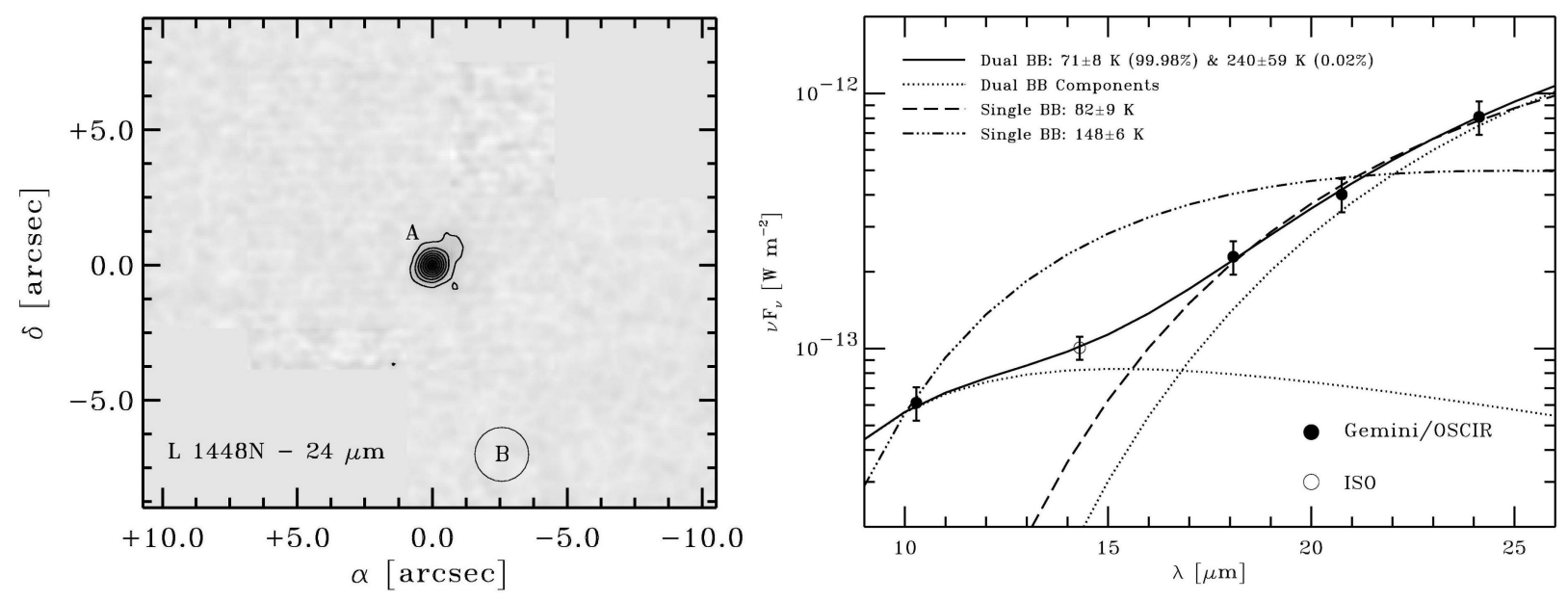

Fig. 6: Gemini imaging of the class 0 source $\mathrm{L} 1448^{11}$. The associated spectral energy distribution is shown at right.

and more complex versions of these systems are becoming accessible to detailed examination with larger telescopes. It is also evident from the complex spectral energy distribution shown in Fig. 6 that high-angular resolution, multiwavelength imaging is critical to disentangling its significance.

The class of Herbig Ae/Be stars also illustrates well the role that detailed mid-IR imaging in exploring the environments of early-type stars. Herbig Ae/Be stars are considered to be the progenitors of main sequence B and A stars, i.e., the higher-mass $\left(2-10 \mathrm{M}_{\odot}\right)$ analogs to $\mathrm{T}$ Tauri stars, which are pre-main-sequence solar-mass stars. A variety of arguments strongly support the idea that the excess IR emission from T Tauri stars originates in circumstellar disks. By analogy, then, it has seemed reasonable to make the same assumption about the excess IR emission in Herbig Ae/Be stars. Detailed mid-infrared imaging and spectroscopic studies of the class of Herbig stars are underway be several groups, and these are clarifying the relative roles of disks and envelopes in generating the IR emission from Herbig Ae/Be stars. For example, Polomski et al. ${ }^{12}$ have imaged the Herbig star AS 310, an early B star. As Fig. 7a shows, complex structure clearly unassociated with a circumstellar disk is evident out to more than $10 \operatorname{arcsec}(25,000 \mathrm{AU})$ from the emission peak, although the compact emission near the peak may arise in an unresolved disk. The compact emission within a 1-arcsec radius of the peak represents only $\sim 12 \%$ of the total $10 \mu \mathrm{m}$ flux in the image, so obviously any attempt to model disk structure for this object must carefully exclude most of the extended emission, which is impossible without these kinds
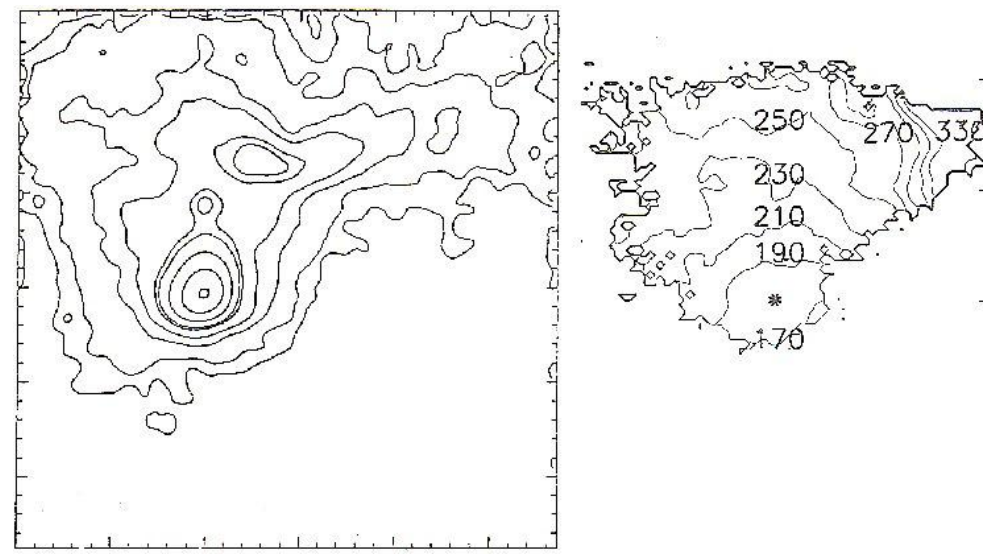

Fig. 7: (a) image at $10 \mu \mathrm{m}$ of the Herbig Ae/Be star AS $310^{12}$ (small tic marks are separated by 1 arcsec). (b) Corresponding distribution of dust temperatures, shown at same scale as image. The star is located at the emission peak and the star symbol. 
of detailed images. Clearly, non-disk emission is generally important in these sources, and must be taken into account in any interpretation of disk properties for this class of star. These types of multi-wavelength images also permit the determination of dust temperatures and optical depths throughout this complex region. The dust temperature distribution, shown in Figures $7 \mathrm{~b}$, indicates that the dust temperature is highest relatively far away from the source, an indication that very small grains may contribute significantly to the mid-IR emission.

\section{ACTIVE GALACTIC NUCLEI}

The full range of mid-infrared techniques can be brought to bear on determining the relative roles of populations of young stars and accretion disks around black holes in generating the often enormous luminosities observed in the centers of many galaxies. Starburst galactic nuclei contain populations of massive stars usually spread over regions extending several hundreds to several thousands of parsecs near the galactic nuclei. In contrast, active galactic nuclei (AGN) are generally thought to be powered by the accretion of matter onto massive black holes. Since that activity is expected to be confined to much smaller regions than are starbursts, determination of the size of the region over which the large luminosities originate can be a crucial piece of information about the true nature of the power source. Mid-infrared imaging can penetrate the often heavy obscuration and establish the size of the source. An excellent example is that of the Seyfert galaxy NGC $5506^{13}$ (Fig. 8). The HST optical image indicates a complex central region several hundred parsecs in size, whereas the mid-infrared image made with MIRLIN at Keck ${ }^{13}$ indicates that the central power source is unresolved (note the diffraction ring) and hence smaller than about $40 \mathrm{pc}$. The unresolved source accounts for about $2 / 3$ of the total mid-infrared emission detected by IRAS. These facts taken together suggest the most of the central power is generated by an accretion disk surrounding a black hole.

The ability of the mid-infrared to address fundamental questions about active galactic nuclei is further demonstrated by recent Gemini imaging of the jet and nucleus of the giant elliptical galaxy M87. Crucial to the so-called unified theories of AGN is the concept of a geometrically and optically thick torus of gas and dust that obscures the accretion disc and black hole. The obscuring torus should re-radiate in the mid-infrared much of the energy it intercepts, thus providing a direct detection method for its existence. It is generally accepted that accretion onto a nuclear black hole with a mass of a few billion solar masses powers the relativistic jet that is seen to extend $25 \operatorname{arcsec}(2 \mathrm{kpc})$ from the nucleus of M87. The jet and nucleus are evident at optical, radio, and x-ray wavelengths, and both features were imaged at Gemini North in the mid-infrared ${ }^{14}$, as shown in Fig. 9. Several of the knots of the jet are clearly detected. The mid-infrared emission detected from these knots appears to be synchrotron radiation. The nuclear emission has a $10 \mu \mathrm{m}$ flux density of $16 \mathrm{mJy}$, and there is marginal evidence for extended emission around the unresolved nucleus. That extended emission, which is $7.3 \%$ of the total nuclear emission, may be associated with the accretion disk, or torus, although this possibility is only speculation at this time. Some models predict that the accretion disk might be expected to have a scale size of $0.3-3$ arcsec, corresponding to $20-200 \mathrm{pc}$. However, using the new mid-infrared flux measurement as well as other pre-

\section{HST}

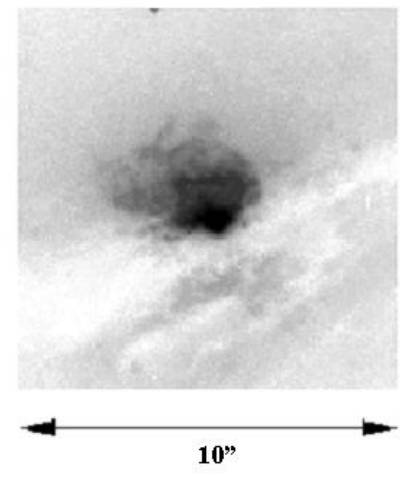

HST central magnitude F606w $=19.41$
MIRLIN

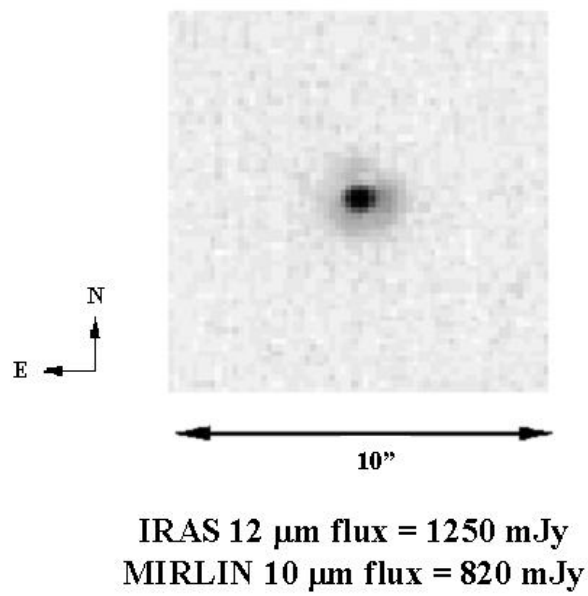

Fig. 8: Comparison of optical and mid-Infrared images of the Seyfert galaxy NGC $5506^{13}$. 

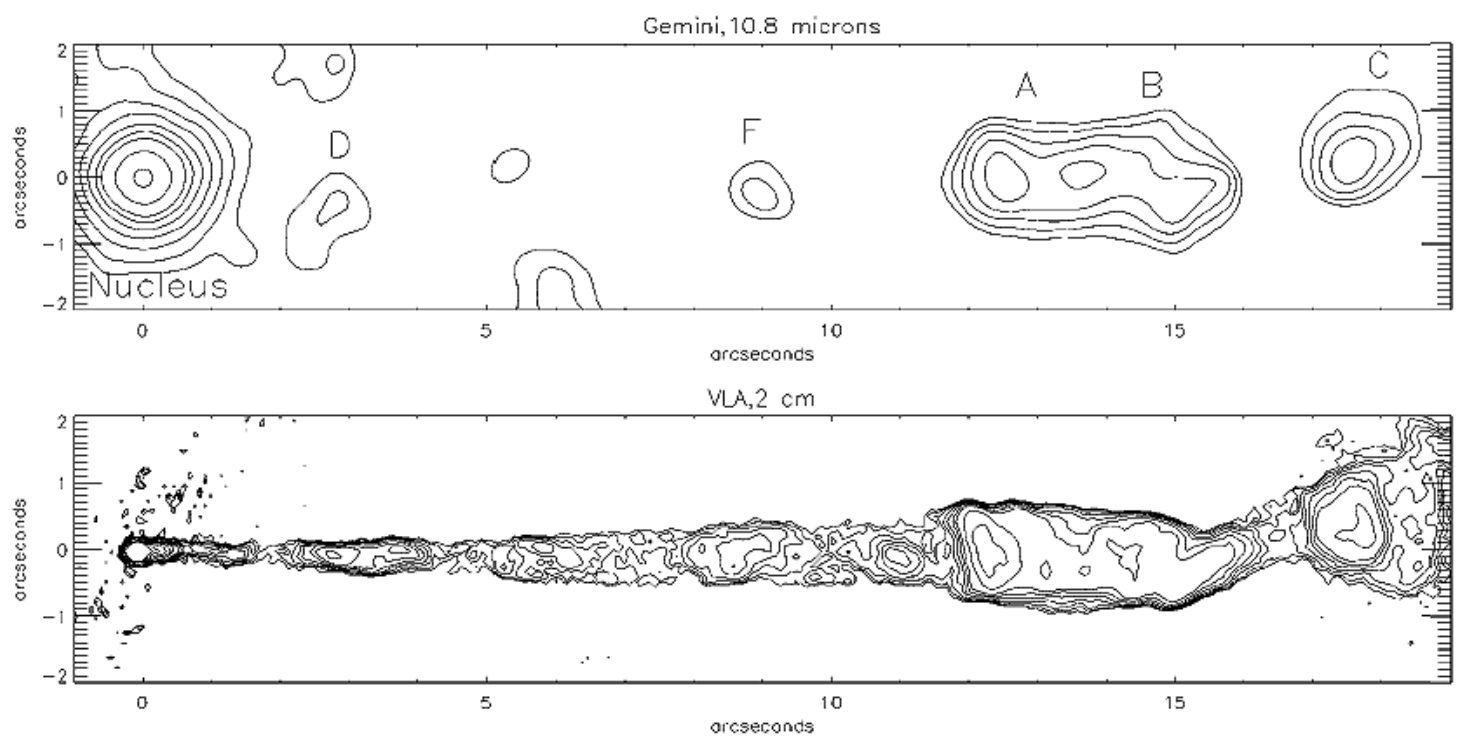

Fig. 9: Comparison of mid-infrared (top) and radio images of nucleus and jet in the active galaxy M87 ${ }^{14}$.

published data, the nuclear flux appears to be dominated by synchrotron emission rather than thermal re-radiation from the torus ${ }^{14,15}$. In fact these observations of the nucleus, which are partly contaminated by thin terrestrial cloud cover and were made with a non-optimised visitor instrument (OSCIR), nevertheless constitute the deepest groundbased midinfrared image ever made, having noise levels near 100 microJanskys. With facility instruments on excellent photometric nights, the 8 and 10-m telescopes should achieve noise levels two or even three times better than this.

Mid-infrared polarimetry is an area that will experience explosive growth as the new generation of mid-infrared instruments is implemented on the largest telescopes. The value of this technique is illustrated by considering the $10 \mu \mathrm{m}$ imaging polarimetry of the nucleus of the Seyfert 2 galaxy NGC $1068^{16}$. These observations, made at the AAT and shown in Fig. 10, have about 1 arcsec resolution. These mid-infrared observations probe the nuclear dust much deeper than do the previous near-infrared and optical observations. They show that the mid-infrared radiation is extended over a

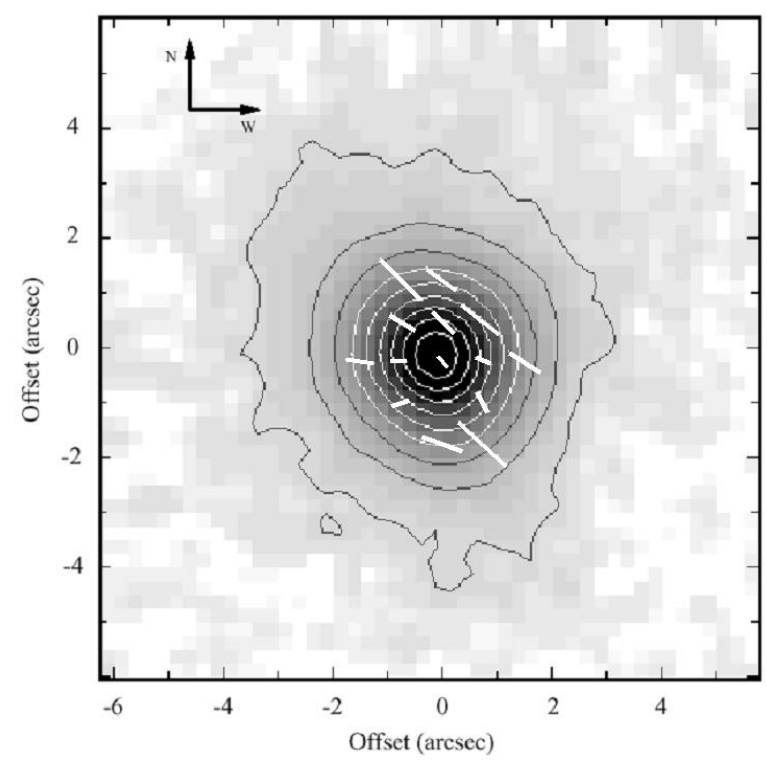

Fig. 10: Mid-infrared polarization map of the nucleus of NGC 1068 made with the instrument NIMPOL at the Anglo-Australian Telescope ${ }^{16}$ 
region several arcseconds in size. Among the conclusions that Lumsden et al. ${ }^{16}$ draw using these data is the fact that scattering from dust, which is the most obvious mechanism, cannot by itself account for the mid- infrared and nearinfrared polarization. They conclude that absorption and reemission by aligned dust grains is the dominant mechanism in the mid-infrared. The warm dust emitting the mid-infrared obscures a region with much hotter dust, which emits mainly in the near-infrared and may be the torus surrounding the accretion disk. The warm, mid-infrared-emitting dust screens the hot dust with the equivalent visual extinction of 20 - 40 magnitudes and may be located in circumnuclearmolecular clouds and cones of outflowing material. The high angular resolution and sensitivity of large telescopes will permit this complex geometry to be probed in better detail, presenting the possibility that the midinfrared-emitting region can be accurately separated from the inner hot-dust region. Among the instruments that are being developed to address these and other issues is CanariCam, which will be used at the GTC. CanariCam has a dualbeam polarimetric mode that permits simultaneous measurement of the ordinary $(o)$ and extraordinary $(e)$ rays. That capability not only increases observational efficiency but also minimizes effects of seeing and changes in atmospheric transparency, increasing the accuracy of the degree of polarization.

\section{REFERENCES}

1. G. Basri, "Observations of brown dwarfs," Ann. Rev. Astron. \& Astrophys. 38, pp. 485-519, 2000.

2. T. Nakajima, B. R. Oppenheimer, S. R. Kulkarni, D. A. Golimowski, K. Matthews, and S. T. Durrance, "Discovery of a cool brown dwarf," Nature 378, pp. 463-465, 1995.

3. M. S. Marley, D. Saumon, T. Guillot, R. S. Freedman, W. B. Hubbard, A. Burrows, and J. I. Lunine, "Atmospheric, Evolutionary, and Spectral Models of the Brown Dwarf Gliese 229B,” Science 272, 1919-1921, 1996.

4. J. D. Kirkpatrick, I. N. Reid, J. Liebert, R. M. Cutri, B. Nelson, C. A. Beichman, C. C. Dahn, D. G. Monet, J. E. Gizis, and M. F. Skrutskie, "Dwarfs cooler than M: the definition of spectral type L using discoveries from the 2micron all-sky survey (2MASS)," Astrophys. J. 519, 802-833, 1999.

5. K. Mathews, T. Nakajima, S. Kulkarni, and B. Oppenheimer, Int. Astron. Union. Circ. 6280, 1995.

6. D. Apai, I. Pascucci, Th. Henning, M. F. Sterzik, R. Klein, D. Semenov, E. Günther, and B. Stecklum, "Probing dust

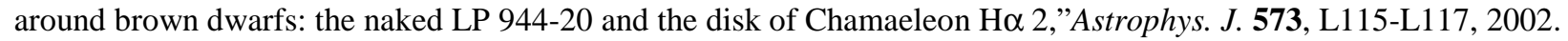

7. R. Jayawardhana, S. Fisher, L. Hartmann, C. Telesco, R. Piña, and G. Fazio, "A dust disk surrounding the young star HR 4796A,” Astrophys. J. 503, L79-L82, 1998.

8. C. M. Telesco, R. S. Fisher, R. K. Piña, R. F. Knacke, S. F. Dermott, M. C., Wyatt, K. Grogan, E. K. Holmes, A. M. Ghez, L. Prato, L. W. Hartmann, and R. Jayawardhana, "Deep 10 and 18 micron imaging of the HR 4796A circumstellar disk: transient dust particles and tentative evidence for a brightness asymmetry," Astrophys. J. 530, 329$341,2000$.

9. R. Fisher, C. Telesco, N. Mariñas, J. Radomski, R. Piña, and T. Hayward, "Imaging of $\beta$ Pictoris in the mid-infrared from Gemini-South," in preparation.

10. L. W. Looney, L. G. Mundy, and W. J. Welch, "Unveiling the circumstellar envelope and disk: a subarcsecond survey of circumstellar structures," Astrophys. J. 529, 477-498, 2000.

11. D. R. Ciardi, J. P. Williams, C. M. Telesco, R. S. Fisher, C. Packham, R. Piña, and J. Radomski, "Mid-infrared imaging of the protostellar binary L1448N-IRS3(A,B)," submitted for publication.

12. E. F. Polomski, C. M. Telesco, R. Piña, and B. Schulz, "The dusty circumstellar environments of Ae/Be Protoplanetary disk candidates," Astron. J., in press (October 2002).

13. V. Gorjian, presented in G. Serabyn, "A mid-infrared camera for the next generation space telescope," Report to NASA, 1999.

14. E. S. Perlman, W. B. Sparks, J. Radomski, C. Packham, R. S. Fisher, R. Piña, and J. A. Biretta, "Deep 10 micron imaging of M87," Astrophys. J. 561, L51-L54, 2001.

15. D. Whysong and R. Antonucci, "A hidden nucleus in Cygnus A, but not in M87," Astrophys. J., submitted (astro$\mathrm{ph} / 0106381), 2001$.

16. S. L. Lumsden, T. J. T. Moore, C. Smith, T. Fujiyoshi, J. Bland-Hawthorn, and M. J. Ward, "Near-and mid-infrared imaging polarimetry of NGC 1068," Mon. Not. R. Astron. Soc. 303, 209-220, 1999.

*telesco@astro.ufl.edu; phone 1352 392-2052, ext. 265; fax 1352 392-5089; www.astro.ufl.edu; Department of Astronomy, University of Florida, 211 Bryant Space Science Center, PO Box 112055, Gainesville, FL USA 32611-2055 